\title{
In Silico Analysis of Functionalized Hydrocarbon Production Using Ehrlich Pathway and Fatty Acid Derivatives in an Endophytic Fungus
}

\author{
Kristopher A. Hunt ${ }^{1,2,+}+\mathbb{D}$, Natasha D. Mallette ${ }^{1,2, \ddagger}$, Brent M. Peyton ${ }^{1,2}$ and Ross P. Carlson $1,2, *$ (D) \\ 1 Center for Biofilm Engineering, Montana State University, Bozeman, MT 59717, USA; \\ hunt0362@uw.edu (K.A.H.); natashamallette@gmail.com (N.D.M.); bpeyton@montana.edu (B.M.P.) \\ 2 Department of Chemical and Biological Engineering, Montana State University, Bozeman, MT 59717, USA \\ * Correspondence: rossc@montana.edu; Tel.: +1-(406)-994-3631 \\ + Current address: Department of Civil and Environmental Engineering, University of Washington, \\ Seattle, WA 98105, USA. \\ $\ddagger$ Current address: Department of Nuclear Science \& Engineering, Oregon State University, \\ Corvallis, OR 97331, USA.
}

Citation: Hunt, K.A.; Mallette, N.D.; Peyton, B.M.; Carlson, R.P. In Silico Analysis of Functionalized Hydrocarbon Production Using Ehrlich Pathway and Fatty Acid Derivatives in an Endophytic Fungus. J. Fungi 2021, 7, 435. https:// doi.org/10.3390/jof7060435

Academic Editor: Laurent Dufossé

Received: 30 April 2021

Accepted: 26 May 2021

Published: 29 May 2021

Publisher's Note: MDPI stays neutral with regard to jurisdictional claims in published maps and institutional affiliations.

Copyright: (c) 2021 by the authors. Licensee MDPI, Basel, Switzerland. This article is an open access article distributed under the terms and conditions of the Creative Commons Attribution (CC BY) license (https:/ / creativecommons.org/licenses/by/ $4.0 /)$.

\begin{abstract}
Functionalized hydrocarbons have various ecological and industrial uses, from signaling molecules and antifungal/antibacterial agents to fuels and specialty chemicals. The potential to produce functionalized hydrocarbons using the cellulolytic, endophytic fungus, Ascocoryne sarcoides, was quantified using genome-enabled, stoichiometric modeling. In silico analysis identified available routes to produce these hydrocarbons, including both anabolic- and catabolic-associated strategies, and determined correlations between the type and size of the hydrocarbons and culturing conditions. The analysis quantified the limits of the wild-type metabolic network to produce functionalized hydrocarbons from cellulose-based substrates and identified metabolic engineering targets, including cellobiose phosphorylase (CP) and cytosolic pyruvate dehydrogenase complex (PDHcyt). CP and PDHcyt activity increased the theoretical production limits under anoxic conditions where less energy was extracted from the substrate. The incorporation of both engineering targets resulted in near-complete conservation of substrate electrons in functionalized hydrocarbons. The in silico framework was integrated with in vitro fungal batch growth experiments to support $\mathrm{O}_{2}$ limitation and functionalized hydrocarbon production predictions. The metabolic reconstruction of this endophytic filamentous fungus describes pathways for both specific and general production strategies of 161 functionalized hydrocarbons applicable to many eukaryotic hosts.
\end{abstract}

Keywords: endophyte; consolidated bioprocessing; efma; fba; cytosolic pyruvate dehydrogenase

\section{Introduction}

Ascocoryne sarcoides is an endophytic fungus isolated from the Eucryphia cordifolia tree in Chilean Northern Patagonia and has been studied for its capacity to produce functionalized hydrocarbons (FHs) [1-6]. Endophytes are ubiquitous components of plant microbiomes that may contribute advantageous and essential functions to their plant host, such as tolerance to nutrient, water, temperature, and salinity stresses, as well as protective strategies against predation [7]. A. sarcoides produces a variety of FHs hypothesized to play a role in the symbiotic relationship with its host [8,9]. FHs produced by $A$. sarcoides also have uses as renewable chemicals, such as biofuels and flavor compounds, made from low-cost substrates, including cellulose and other agricultural wastes [10-12]. This consolidation of $\mathrm{FH}$ production from a diverse range of feedstocks makes $A$. sarcoides a candidate for bioprocessing [13]. The production of FHs by A. sarcoides has been studied in vitro at the organism level, but there have been minimal molecular-level pathway examinations and no in silico reconstructions of its metabolism [3]. 
Production of FHs has been shown to occur via four primary metabolic routes in eukaryotes: traditional fermentation, fatty acid production, linoleic acid degradation, and Ehrlich pathways. Traditional fermentation pathways produce low molecular weight byproducts from central metabolisms, such as ethanol and acetate. Fatty acid biosynthetic machinery can produce many FHs as a function of the initiating molecule, the number of elongation cycles, and endpoint of the final cycle. Examples include octane, octanol, and hexanoic acid [14]. Linoleic acid degradation can produce a variety of $\mathrm{C}_{8}$ to $\mathrm{C}_{10}$ FHs, such as 1-octen-3-ol, through the oxidation of double bonds and subsequent redox reactions [3]. Ehrlich pathways can produce various $C_{4}$ to $C_{10}$ FHs through the deamination and decarboxylation of amino acids or their derivatives, including 1-methyl-3-butanol [15]. Additionally, alcohols and acids from the described routes can be used to produce esters, further expanding the range of possible FHs $[14,16,17]$. A thorough, systems-based analysis of these metabolic strategies in an endophytic fungus has not been reported.

Acetyl-CoA is a building block for many FHs and their precursors, such as lipids and some amino acids [18]. The production and degradation of fatty acids in eukaryotes are functionally partitioned using acyl carrier protein (ACP) and coenzyme A (CoA). Production and degradation are also spatially partitioned through the localization of enzymes between the cytosol, mitochondria, and peroxisome [19]. Metabolite compartmentalization creates control points for balancing cellular energy generation in the mitochondria and fatty acid production in the cytosol. Acetyl-CoA can be transported between the mitochondria and the cytosol using three routes, with each having different cellular energy requirements (Figure 1) [20]. The ATP-citrate lyase (Acl) route cleaves citrate produced in the mitochondria into oxaloacetate and acetyl-CoA in the cytosol [21-23]. The acetate route converts acetyl-CoA to acetate in the mitochondria, transports the acetate to the cytosol, and reactivates it via acetyl-CoA synthetase (Acs). The acyl-carnitine route uses acyl-carnitine transport to move acyl groups between the cellular compartments for $\beta$-oxidation. Still, it cannot be the sole route for transporting acetyl-CoA from mitochondria to the cytosol without genetic engineering $[18,23]$. The pathways used to generate acetyl-CoA typically correlate with the metabolic strategy of the organism. Oleaginous yeasts generate most of their acetyl-CoA in the cytosol using the Acl enzyme; the citrate used to form acetyl-CoA originates from mitochondria and must be transported into the cytosol. Non-oleaginous yeasts generate most of their acetyl-CoA in the cytosol through pyruvate decarboxylase (Pdc), acetaldehyde oxidase, and Acs activities, a process that does not require organelle transport processes (Figure 1) [18].

Stoichiometric modeling is a computational systems biology approach that can identify ecologically relevant phenotypes and gene targets for optimizing metabolic network function. It does so by accounting for critical central metabolism properties, including enzyme compartmentalization and cellular energy costs for metabolite transport [24-27]. There are two primary forms of stoichiometric modeling; both require an initial representation of the metabolic network, commonly generated from genomic or other experimental data [28-31]. The metabolic network is represented as a set of linear equations that define genome enabled and abiotic metabolite conversions. Flux balance analysis (FBA) uses these linear equations, bounds on reaction rates, and an objective function (i.e., maximizing biomass production) to obtain an optimal flux distribution [32]. Elementary flux mode analysis (EFMA) uses the same set of linear equations to obtain the smallest set of genetically distinct flux distributions known as elementary flux modes (EFMs). The complete set of EFMs represents all possible steady-state metabolisms of the network through nonnegative linear combinations allowing for predictions of maximum yields and the effect of genetic modifications [28,33,34].

Fungal endophytes are an important natural component of plant microbiomes and potential consolidated bioprocess catalysts. The presented work is one of the first metabolic reconstructions of an endophytic fungus, with the first study examining production of therapeutic resveratrol [35], and maps both specific and general FH production strategies applicable to many eukaryotes. The results compare FH production from the carbon con- 
serving Ehrlich pathway and electron concentrating fatty acid derivatives with respect to substrate carbon and electrons conservation, chain length, and functional groups. Additionally, the results quantify the stoichiometric limitations of FH production with respect to (i) the theoretically optimal chain length of products from cellulose, (ii) the theoretical limits of cellulosic feedstocks considering metabolic engineering targets, and (iii) the impact of culturing conditions on metabolite profiles. Comparison of theoretical limits with observed production trends provides the foundation for FH production with applicability to biotechnology and biochemical analysis of eukaryotic compartmentalization.



Figure 1. Overview of fungal acetyl-CoA metabolism. Common acetyl-CoA pathways in fungi are shown in solid arrows. A cytosolic pyruvate dehydrogenase (heavy dashed line) may increase functionalized hydrocarbon production but was not found in fungi and was not annotated in Ascocoryne sarcoides NRRL 50072. Enzyme abbreviations: ACH-acetyl-CoA hydrolase, Acl-ATP citrate lyase, Acs-acetyl-CoA synthetase, ACT—acylcarnitine transport, Ald-aldehyde dehydrogenase, $\mathrm{CP}$ - cellobiose phosphorylase, $\mathrm{Pdh}_{\text {cyt }}$ - pyruvate dehydrogenase, $\mathrm{Pdh}_{\text {cyt }}$ - cytosolic pyruvate dehydrogenase, Pdc-pyruvate decarboxylase.

\section{Materials and Methods}

\subsection{Construction of Fungal Endophyte Metabolic Model}

An A. sarcoides NRRL 50072 metabolic model was constructed from the genome annotations and transcriptome data available at http://asco.gersteinlab.org (accessed on 30 April 2021) [3]; incomplete pathways were filled using assumed reactions based on completeness of a pathway and reference organisms (Supplementary Materials). Enzyme localization was modeled based on predictions from TargetP 1.1 [36] and considered for two compartments, the mitochondria and cytosol. The macromolecular composition of 
biomass was modeled after Thielavia terrestris as a representative filamentous fungus [37]. Protein and RNA monomer composition was determined by the average distribution of all open reading frames in the genome and the ribosomal subunits, respectively. DNA monomer composition was modeled to represent the guanine-cytosine content of the genome. Micronutrients were assumed non-limiting and nitrogen supplied by ammonium, sulfur by sulfate, and phosphorous by phosphate following published $A$. sarcoides NRRL 50072 metabolite studies [1-4]. $\mathrm{O}_{2}$ was required to produce essential fatty acids and, therefore, biomass.

\subsection{Stoichiometric Analyses}

The stoichiometric model was compiled as described in the following section using Microsoft EXCEL and converted to appropriate formats using CellNetAnalyzer version $2014.1[38,39]$. Flux distributions were enumerated using either RegEFMTool version 2.0 [40] or the constraints-based reconstruction and analysis (COBRA) toolbox [41] run in MATLAB version 2016B. Gene regulatory rules excluding the simultaneous use of mutually exclusive reactions were applied to minimize the number of physiologically irrelevant possibilities (Supplementary Materials). All computations were performed on a machine with a maximum of 2 Intel Xeon X5690s and 120 GB of memory.

\section{Results}

3.1. In Silico Reconstruction of Carbon Uptake, Compartmentalization, and Functionalized Hydrocarbon Production

The nutrient requirements to produce cellular energy and functionalized hydrocarbons (FHs) in A. sarcoides were influenced by oligosaccharide chain length and acetyl-CoA transport routes. A. sarcoides can utilize cellulose as a carbon substrate [5], which has been shown to be degraded to soluble oligosaccharides (i.e., $1-5$ glucose units) extracellularly in other fungi [42]. The impact of oligosaccharide chain length on cellular energetics was quantified by modeling the representative carbon and electron sources glucose and cellopentaose. These substrates enter the cell through $\mathrm{ABC}$ transporters, the oligosaccharides are depolymerized independent of phosphate, and a hexose kinase activates monosaccharides. Production of cytosolic acetyl-CoA was modeled according to the annotated genes for the Acl and Acetate routes (Figure 1). A Pdc enzyme was not found in the $A$. sarcoides genome, and native acyl-carnitine transport is not expected to be a major route for acetyl-CoA transport from mitochondria to the cytosol [18,23].

Metabolic model reactions for $\mathrm{FH}$ production were built using genomic and metabolomic data. A consensus list of FHs was assembled by collating any FH species reported by at least three publications about $A$. sarcoides (Table 1). These FHs were modeled using traditional fermentation, fatty acid production, linoleic acid degradation, and Ehrlich pathways (Figure 2). Fatty acid pathways can produce a wide variety of FHs subject to the initiating molecule (e.g., acetyl-CoA or propanoyl-CoA), monomers incorporated during elongation (e.g., $C_{2}$ and $C_{3}$ monomers), and stage of the final reduction cycle. These variations in components and pathways have a combinatorial effect $[14,43,44]$ and explain most FHs reported for $A$. sarcoides (Table 1). The metabolic model considered acetyl- and propanoyl-CoA as the initiators for alkyl chains, while only acetyl-CoA was considered an elongating monomer to avoid excessive computational complexity. This approach resulted in even- or odd-chained FHs depending on the initiator molecule, acetyl-CoA or propanoyl$\mathrm{CoA}$, and the stage of fatty acid elongation (Figure 2). Esterification of FHs from these pathways accounted for esters reported in vitro (Table 1). In total, 161 different excreted FHs composed of eight different functional groups ranging from $C_{3}$ to $C_{20}$ were modeled. 
Table 1. Commonly reported functionalized hydrocarbons from A. sarcoides NRRL $50072{ }^{a}$ organized by hypothesized production pathway.

\begin{tabular}{ccc}
\hline Fatty Acid Derivatives & Ehrlich Pathway Derivatives & Linoleic Acid Derivatives \\
\hline 1-butanol & 2-methyl-1-propyl acid, alcohol, & 3-octanone \\
1-hexanol & and esters & 1-octen-3-ol \\
Hexanoate & 3-methyl-1-butyl acid, alcohol, & \\
1-heptanol & and esters & Miscellaneous \\
Octane & 2-phenylethyl alcohol and esters & $\begin{array}{c}\text { Propyl-cyclopropane } \\
\text { 2-nonanone }\end{array}$ \\
$\mathrm{C}_{2}, \mathrm{C}_{5}-\mathrm{C}_{10}$ alkyl acetates & Phenyl methanol & 2-pentyl-furan ${ }^{\mathrm{b}}$ \\
\hline
\end{tabular}

a All functionalized hydrocarbons were reported from at least three separate sources [1-3,5, and this study]; ${ }^{\mathrm{b}}$ Not included in the model.

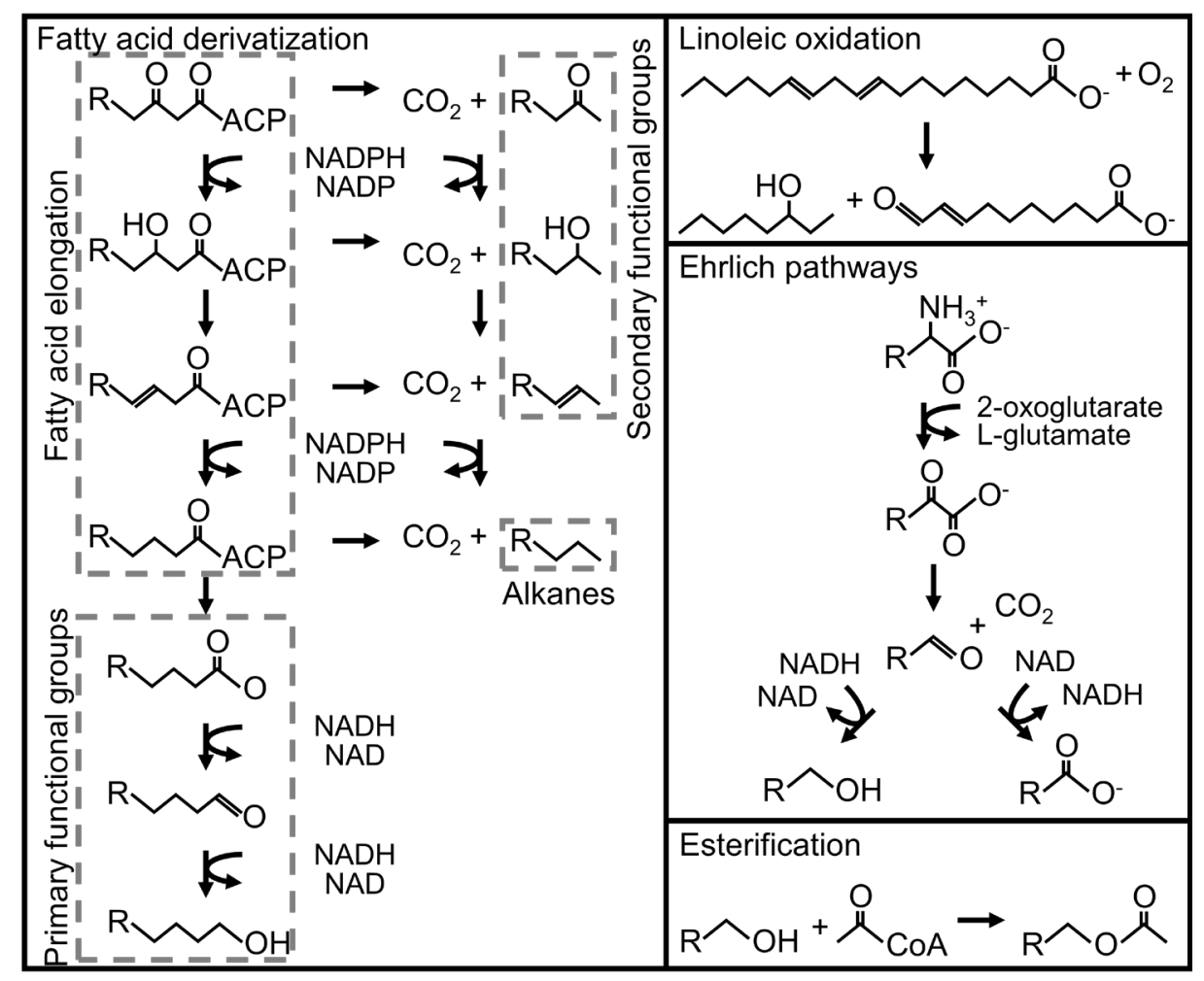

Figure 2. Overview of functionalized hydrocarbon producing pathways relevant to $A$. sarcoides NRRL 50072. Three pathways are depicted: fatty acid derivatization, linoleic oxidation, and Ehrlich pathways $[3,14,15,43]$. Products from these pathways and their esters account for most observed FHs. $\mathrm{ACP}$ refers to the acyl carrier protein associated with fatty acid synthesis, while $\mathrm{R}$ refers to carbon backbones. Associated genetic annotations can be found in the Supplementary Materials.

\subsection{In Silico Analysis of Cellular Energy Production}

Ecologically competitive phenotypes for cellular energy production were identified using resource allocation theory, postulating that the fittest cellular phenotypes minimize the amount of limiting resource required to make a product [45]. The theory was applied by calculating the carbon source and $\mathrm{O}_{2}$ required to produce cellular energy for each elementary flux mode (EFM) as defined by the Cmoles of carbon source and moles of $\mathrm{O}_{2}$, respectively, consumed to produce a mole of cellular energy (equal to one mole of ATP phosphodiester bonds). EFMs that minimized carbon and $\mathrm{O}_{2}$ required to produce cellular energy formed a trade-off surface (black line in Figure 3). EFMs that minimized the carbon required to produce cellular energy are to the left in the plot. The least carbon required to produce energy was achieved by metabolic strategies that completely oxidized cellulose to carbon dioxide and water and required 0.2 Cmoles of cellopentaose and 0.2 moles of 
$\mathrm{O}_{2}$ per mole of cellular energy. Under $\mathrm{O}_{2}$ limited conditions, phenotypes that secrete reduced carbon byproducts, such as acetate, acetaldehyde, and ultimately ethanol, became competitive (i.e., moving from the top left to the bottom right of the trade-off surface in Figure 3). Anoxic cellular energy production was predicted (1.3 Cmole cellopentaose per mole of cellular energy), but not anoxic growth due to an $\mathrm{O}_{2}$ requirement to produce sterols and other lipids (Supplementary Materials). A Cmole of cellopentaose produced more cellular energy than a Cmole of glucose due to the modeled sugar transporters. Transport of one cellopentaose (i.e., five monomers of glucose) or one glucose into the cell required the same energy expenditure.
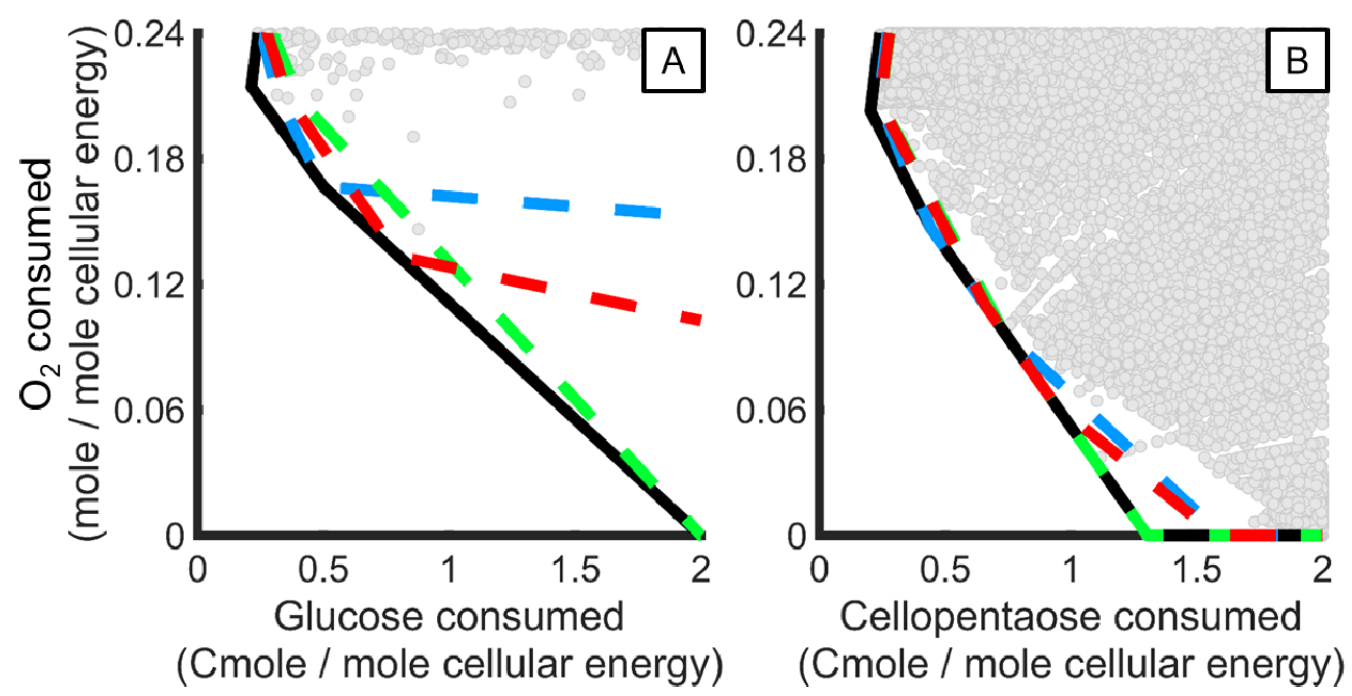

Figure 3. Metabolic strategies for cellular energy production from glucose (A) and cellopentaose (B) that minimize resource utilization span between complete oxidation and fermentation. Overall optimal $\mathrm{O}_{2}$ and carbon source utilization strategies for cellular energy production are highlighted with black boundaries. All possible metabolic strategies (elementary flux modes) are grey points. Metabolic strategies that produce only acetate (blue dashed line), acetaldehyde (red dashed line), or ethanol (green dashed line) are highlighted.

\subsection{In Silico Analysis of Functionalized Hydrocarbon Production}

FH production balances cellular energy production, electron trafficking, and resource utilization, which are all sensitive to nutrient limitations during cultivation. Trade-offs between carbon source and $\mathrm{O}_{2}$ requirements quantified competitive $\mathrm{FH}$ producing phenotypes. The carbon and $\mathrm{O}_{2}$ requirements to produce $\mathrm{FHs}$ were defined as the Cmoles of carbon source or moles of $\mathrm{O}_{2}$, respectively, consumed to make a Cmole of FH. Eight hydrocarbon functional groups (i.e., acids, esters, aldehydes, ketones, primary and secondary alcohols, alkanes, and alkenes) with chain lengths from $C_{3}$ to $C_{20}$ were modeled that represent most FHs reported for $A$. sarcoides (Table 2). FH producing metabolic strategies that required the least carbon source and $\mathrm{O}_{2}$ are to the left and bottom, respectively (Figures 4 and 5). Metabolic strategies that produced a single, target $\mathrm{FH}$ of a specific chain length while minimizing carbon and $\mathrm{O}_{2}$ requirements are depicted by colored lines in Figures 4 and 5. FHs generally required less carbon source to produce in the presence of $\mathrm{O}_{2}$ as quantified by the lowest Cmole of substrate per Cmole of $\mathrm{FH}$ (i.e., negative slopes of colored lines in Figures 4 and 5). Production of Ehrlich pathway derivatives generally required fewer Cmoles of carbon source than fatty acid derivatives. The low molecular weight primary fermentation products formate, acetate, acetaldehyde, and ethanol were not considered as they were not the focus of this study. 
Table 2. Theoretical carbon source and $\mathrm{O}_{2}$ requirements to produce functionalized hydrocarbons based on wild-type $A$. sarcoides NRRL 50072 genomic potential and recombinant strains.

\begin{tabular}{|c|c|c|c|c|c|c|c|c|}
\hline \multirow[b]{2}{*}{ Functional group ${ }^{b}$} & \multicolumn{2}{|c|}{ Wild-type (WT) } & \multicolumn{2}{|c|}{$\mathrm{WT}+\mathrm{CP}^{\mathrm{a}}$} & \multicolumn{2}{|c|}{$\mathrm{WT}+\mathrm{PDH}_{\mathrm{cyt}}{ }^{\mathrm{a}}$} & \multicolumn{2}{|c|}{$\mathrm{WT}+\mathrm{CP}+\mathrm{PDH}_{\mathrm{cyt}}{ }^{\mathrm{a}}$} \\
\hline & Carbon $^{\mathrm{c}}$ & $\mathrm{O}_{2}{ }^{\mathrm{c}}$ & Carbon $^{\mathrm{c}}$ & $\mathrm{O}_{2}{ }^{\mathrm{c}}$ & Carbon $^{\mathrm{c}}$ & $\mathrm{O}_{2}{ }^{\mathrm{c}}$ & Carbon $^{\mathrm{c}}$ & $\mathrm{O}_{2}{ }^{\mathrm{c}}$ \\
\hline Acids & $1.17(1.2)$ & 0.00 & $1.15(1.2)$ & 0.03 & $1.16(1.2)$ & 0.003 & $1.15(1.2)$ & 0.03 \\
\hline Aldehydes & $1.25(1.3)$ & 0.13 & $1.23(1.3)$ & 0.08 & $1.25(1.3)$ & 0.13 & $1.23(1.3)$ & 0.08 \\
\hline Esters & 1.29 & 0 & 1.29 & 0 & 1.29 & 0 & 1.29 & 0 \\
\hline Primary alcohols & $1.31(1.5)$ & 0.12 & $1.28(1.5)$ & 0.09 & $1.31(1.5)$ & 0.12 & $1.28(1.5)$ & 0.09 \\
\hline Ketones & $1.5(2.7)$ & 0.12 & $1.5(2.2)$ & 0.08 & $1.5(2.0)$ & 0.08 & $1.5(1.7)$ & 0.08 \\
\hline Alkenes & $1.62(2.4)$ & 0.12 & $1.57(2.0)$ & 0.07 & $1.52(1.7)$ & 0.02 & 1.5 & 0 \\
\hline Secondary alcohols & $1.62(2.4)$ & 0.12 & $1.57(2.0)$ & 0.07 & $1.52(1.7)$ & 0.02 & 1.5 & 0 \\
\hline Alkanes & $1.64(2.4)$ & 0.11 & $1.60(2.0)$ & 0.07 & $1.54(1.6)$ & 0.01 & 1.53 & 0 \\
\hline
\end{tabular}

${ }^{\text {a }}$ Recombinant strains examined the effect of expressing cellobiose phosphorylase $(\mathrm{CP})$ and a cytosolic pyruvate dehydrogenase complex $\left(\mathrm{PDH}_{\mathrm{cyt}}\right)$.

${ }^{b}$ Each functional group was simulated in isolation. ${ }^{c}$ Carbon and $\mathrm{O}_{2}$ are the Cmoles of cellopentaose and moles of $\mathrm{O}_{2}$, respectively, consumed per

Cmole of functionalized hydrocarbons produced under carbon- or $\mathrm{O}_{2}$-limited conditions.

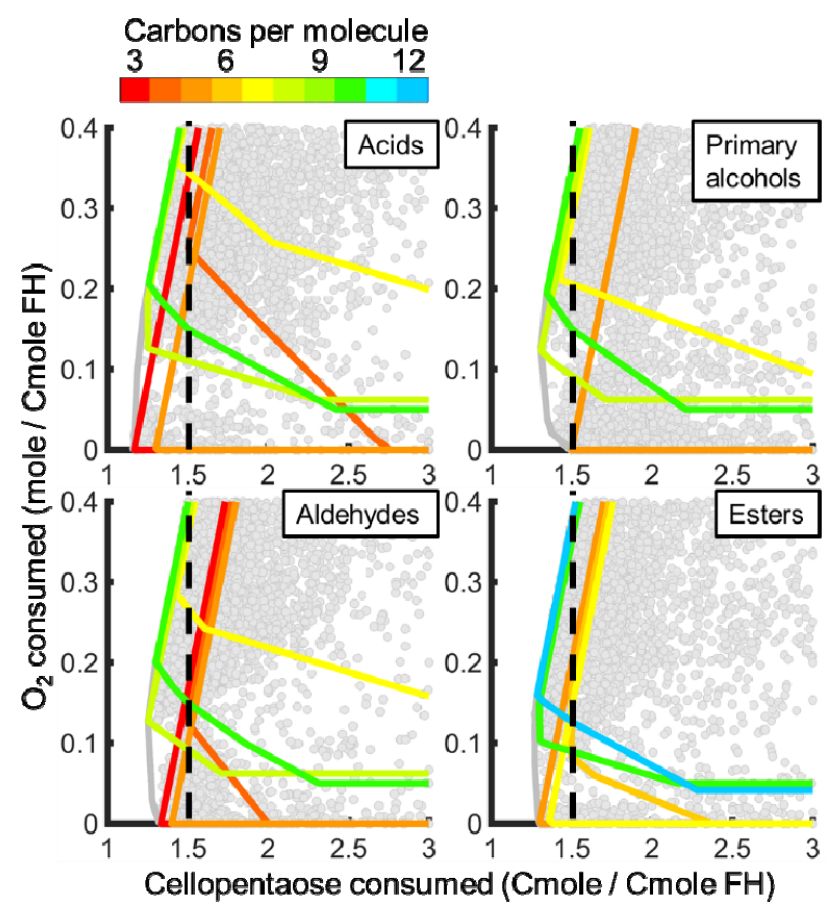

Figure 4. Ehrlich pathway derivative production strategies that minimize carbon and $\mathrm{O}_{2}$ requirements in $A$. sarcoides NRRL 50072 with respect to chain length and defining functional group. Metabolic strategies that minimize carbon source and $\mathrm{O}_{2}$ requirements to produce functionalized hydrocarbons (FHs) were identified (grey boundary, all possible metabolic strategies, elementary flux modes, are grey points). The colored lines denote metabolic strategies that produce FHs with a given number of carbons per molecule. Formate, acetate, acetaldehyde, and ethanol were not considered to be FHs for this study. Profiles for propanol, isobutyl alcohol, and isopentyl alcohol overlap. A reference carbon resource cost of $1.5 \mathrm{Cmole}$ per $\mathrm{Cmole}$ of $\mathrm{FH}$ is denoted by the vertical dashed line to facilitate comparisons. 


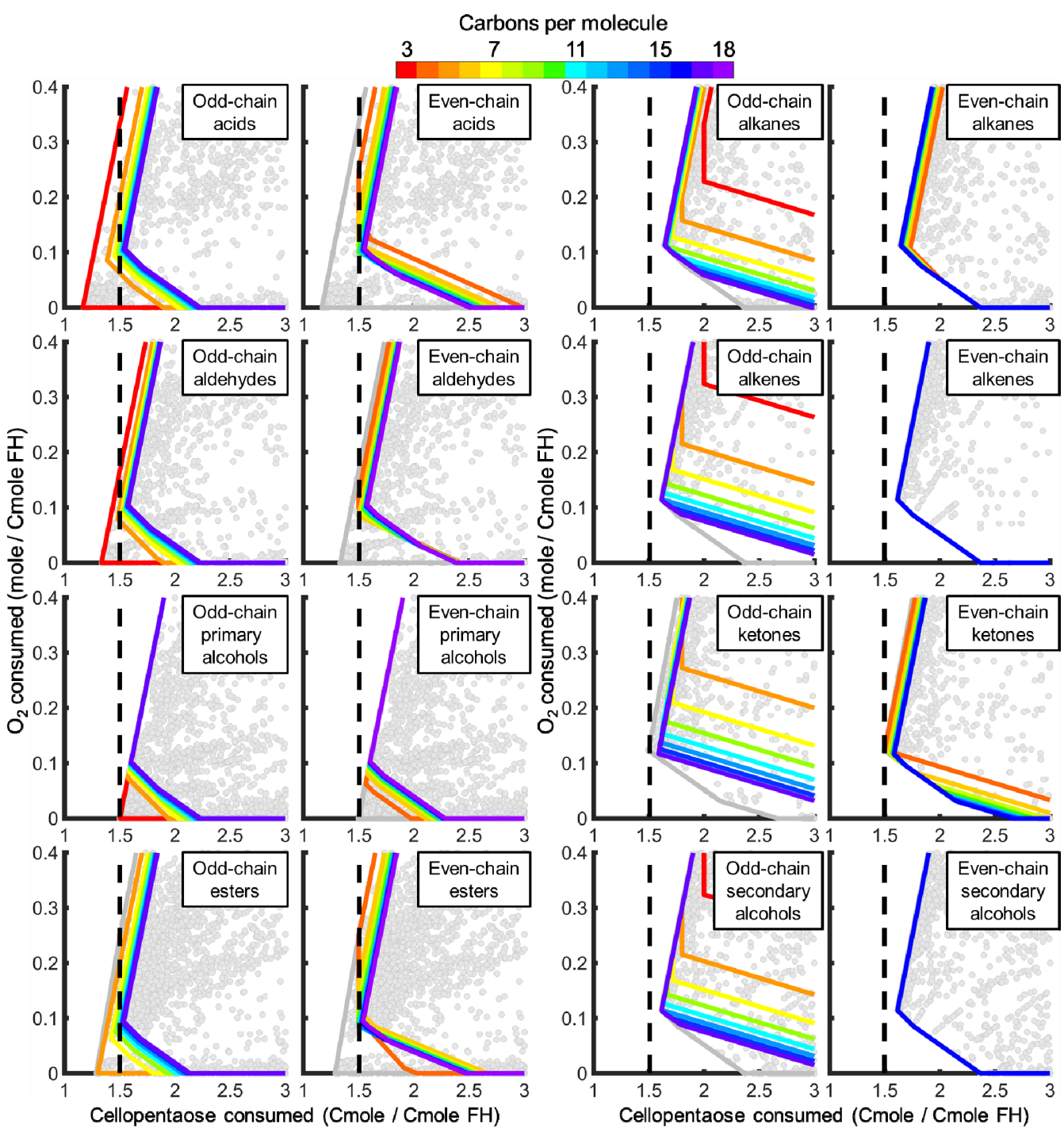

Figure 5. Fatty acid derivative production strategies that minimize carbon and $\mathrm{O}_{2}$ consumption in $A$. sarcoides NRRL 50072. Optimal $\mathrm{O}_{2}$ and carbon source utilization strategies that produce functionalized hydrocarbons (FHs) were identified to minimize resource costs (grey boundary, all possible metabolic strategies, elementary flux modes, and grey points). The colored lines denote metabolic strategies that produce FHs with a given number of carbons per molecule. Formate, acetate, acetaldehyde, and ethanol were not considered to be FHs for this study. Odd and even chain primary functional groups were initiated by propanoyl-CoA and acetyl-CoA, respectively, while the inverse is true for secondary functional groups. Resource profiles for even chain alkanes, even chain secondary alcohols appear as lines due to overlap. A reference carbon resource cost of 1.5 Cmole per Cmole of $\mathrm{FH}$ is denoted by the vertical dashed line to facilitate comparisons.

\subsubsection{Ehrlich Pathway Derivatives}

Resource requirements to produce Ehrlich pathway derivatives from 7 amino acids (i.e., methionine, valine, isoleucine, leucine, phenylalanine, tryptophan, and tyrosine) were governed under oxic conditions by the FH degree of reduction. Aromatic Ehrlich pathway derivatives, such as indole-3-acetate and 4-hydroxyphenylacetate, required the least carbon source of any considered FHs under oxic conditions (green lines in Figure 4), only propanoic acid required less under anoxic conditions (red line in Figure 4). These FHs retained a large fraction of the carbon from the carbon source and have moderate degrees of reduction that clustered around 4.5 (Figure 6). Conversely, Ehrlich pathway derivatives with higher degrees of reduction (i.e., 5-6 electron moles $\mathrm{Cmole}^{-1}$ ), such as isopentyl derivatives, retained less carbon source carbon (orange lines in panels for acids, 
aldehydes, and primary alcohols, yellow for esters of Figure 4). The trade-off between carbon retention and electron density was a function of electron conservation and substrate electron density, which are major design constraints for synthesizing a FH of interest.
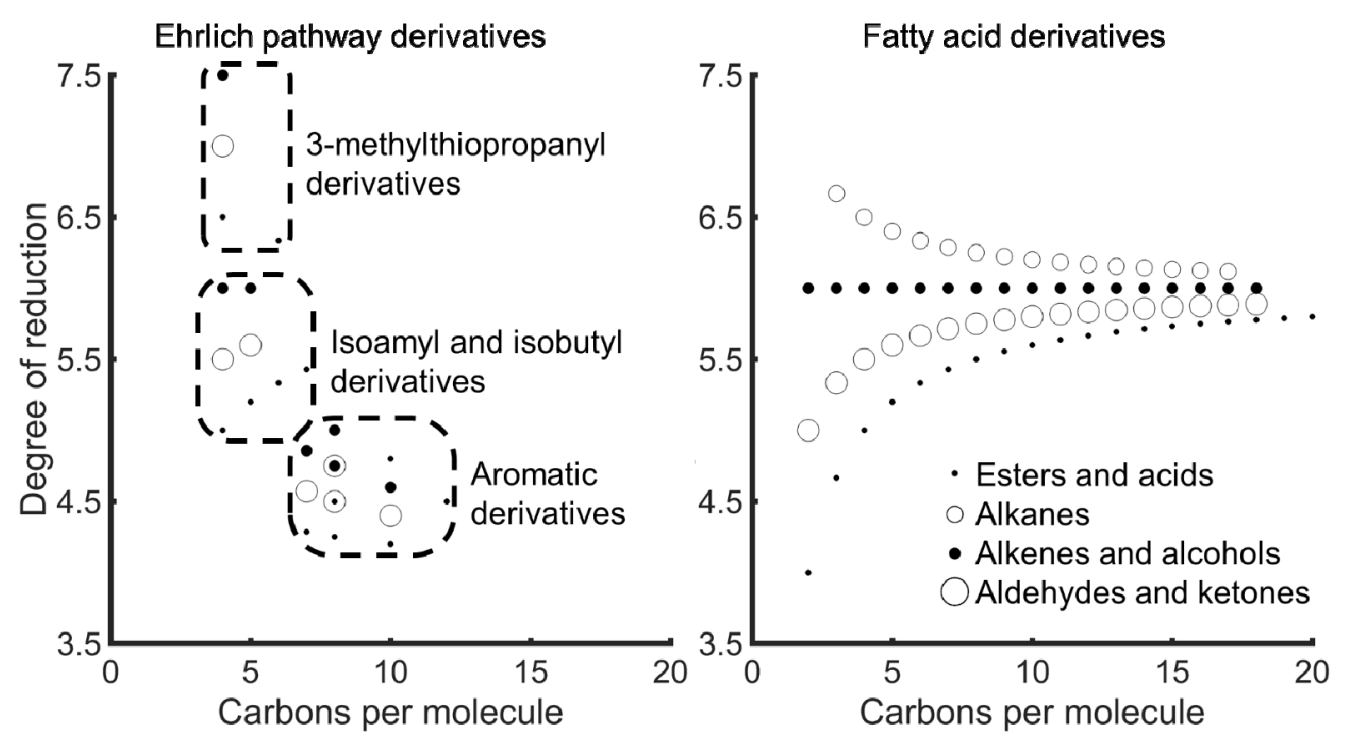

Figure 6. Degree of reduction of functionalized hydrocarbons plotted versus required carbons per molecule.

Simulated production of Ehrlich pathway derivatives indicated an increase in Cmoles of substrate requirements as $\mathrm{O}_{2}$ availability decreased because more carbon source was oxidized for cellular energy production. Isopentyl derivatives were less sensitive to energy requirements than aromatic derivatives and required less carbon source to produce when $\mathrm{O}_{2}$ was limited. A similar trend was observed across examined functional groups (Figure 4). Aromatic FHs could not be produced under anoxic conditions because only the $\mathrm{O}_{2}$ requiring amine oxidase was found in the genome (i.e., a phenylpyruvate decarboxylase was not annotated in the A. sarcoides genome).

\subsubsection{Fatty Acid Derivatives}

Efficient production of fatty acid derivatives was governed by their monomers and cellular energy requirements. Initiating fatty acids with propanoyl-CoA required less carbon source under oxic conditions than initiating it with acetyl-CoA (Figure 5). Propanoate was produced with high carbon source efficiency from 2-oxobutanoate, a precursor of isoleucine metabolism. However, as acetyl-CoA monomers were added to the propanoyl-CoA initiated molecule, the resulting FHs approached the theoretical limit of 1.5 Cmole of carbon source per Cmole of FH produced (Odd chain length acids in Figure 5). In contrast, acetylCoA initiated FHs were already at the theoretical limit independent of chain length (Even chain length acids in Figure 5). Fatty acid derived FHs all lost at least one carbon dioxide per monomeric acetyl-CoA but retained most if not all the electrons from the carbon source, as evidenced by the degrees of reduction approaching 6 (Figure 6). A similar trend was observed for all primary functional groups, whereas secondary functional groups were decarboxylated and lost the initial carbon savings associated with propanoyl-CoA. Fatty acid derivatives produced under low $\mathrm{O}_{2}$ availability required coproduction of other byproducts, such as ethanol and acetate, to produce the cellular energy required for their production and therefore required more carbon source (Figure 5). Acetyl-CoA-initiated fatty acids and aldehydes required formate coproduction under anoxic conditions to balance electrons. Therefore, as the functional group became more reduced (i.e., alcohols are more reduced than aldehydes which are more reduced than acids) and consumed additional electrons, the carbon source requirements decreased. Odd chain length fatty acids and aldehydes 
used propanoyl-CoA as the initiating molecule, which consumed the extra electrons and removed the requirement to coproduce formate.

FHs produced from linoleic acid degradation required more resources than those derived from fatty acids directly and could not be produced as the sole reduced byproduct. For example, all $C_{8}$ derivatives were produced with a $C_{9}$ or $C_{10}$ coproduct and were nontrivial compared with other FHs. However, they required more resources than the $\mathrm{C}_{18}$ precursor from which they were derived.

\subsection{In Silico Metabolic Engineering Targets}

In silico models provide a framework for predicting network modifications to enhance catalytic function [20]. Two enzymatic modifications were tested using the presented A. sarcoides model for their capacity to decrease the cellular energy cost of metabolite transport: (1) the incorporation of a cellobiose phosphorylase (CP) and (2) the incorporation of cytoplasmic pyruvate dehydrogenase $\left(\mathrm{PDH}_{\mathrm{cyt}}\right)$.

\subsubsection{Cellobiose Phosphorylase}

In silico analysis of recombinant $\mathrm{CP}$ activity demonstrated a reduced cellular energy requirement for sugar activation. $\mathrm{CP}$ conserved some of the energy in a $\beta(1,4)$ glucose bond via sugar phosphorylation using inorganic phosphate. The energy conservation alleviated the cost of activating $n-1$ glucose molecules using ATP, where $n$ is the number of monomers in the oligosaccharide being depolymerized. CP activity decreased the predicted requirements for carbon source and $\mathrm{O}_{2}$ for all FHs, except for 2-methyl-propanoate and 2-methyl-butanoate, which did not require a net input of energy to produce (Figure 7). This effect was most prominent under anoxic conditions where cellular energy production is more substrate intensive (Table 2). The Cmoles of substrate required to produce FHs decreased by as much as $17 \%$ when cellopentaose was the carbon source. No change was predicted for cultures grown with glucose $(n=1)$ as the monomeric carbon source does not have $\beta(1,4)$ glucose bonds.

\subsubsection{Cytosolic Pyruvate Dehydrogenase}

Transport of acetyl-CoA from the mitochondria to the cytosol constrains cytosolic production of fatty acids [23]. All acetyl-CoA transport routes described above require energy consumption to transfer mitochondria-derived acetyl-CoA to the cytosol, where it can be incorporated into fatty acids. $\mathrm{PDH}_{\text {cyt }}$ activity was hypothesized to uncouple cytosolic acetyl-CoA production from the energetic transport requirements and provide cytosolic reducing equivalents that increased the yield of fatty acid-derived FHs (dashed green line in Figure 1). This effect was most prominent in anoxic simulations, where the carbon source requirement for $\mathrm{FH}$ production decreased up to $30 \%$. $\mathrm{PDH}_{\text {cyt }}$ activity had a less significant impact under oxic conditions $(6 \%$ decrease in carbon source requirement, Table 2) due to the higher cellular energy yields per carbon source during $\mathrm{O}_{2}$ respiration (Figure 7). $\mathrm{PDH}_{\mathrm{cyt}}$ activity did not impact Ehrlich pathway derivatives because they were produced in the mitochondria when derived from acetyl-CoA. The $\mathrm{PDH}_{\text {cyt }}$-based decreases in substrate requirements for $\mathrm{FH}$ production were similar for both cellopentaose and glucose.

3.4.3. Concurrent Cellobiose Phosphorylase and Cytosolic Pyruvate Dehydrogenase Activity

The concurrent effect of both enzyme modifications decreased the requirement for carbon sources to produce FHs by up to 7 and 38\% under oxic and anoxic conditions, respectively (Table 2). The largest reductions were observed for fatty acid derivatives as they are the most energy intensive and dependent on cytosolic acetyl-CoA. However, fatty acid derivatives were still constrained by a minimum theoretical limit of $1.5 \mathrm{Cmoles}$ carbon source per Cmole of product due to the loss of carbon dioxide during the formation of acetyl-CoA from malonyl-CoA and due to electron conservation between carbon source 
and product. Exceptions to this are fatty acids derived from alternative initiating molecules, such as propanoyl-CoA.

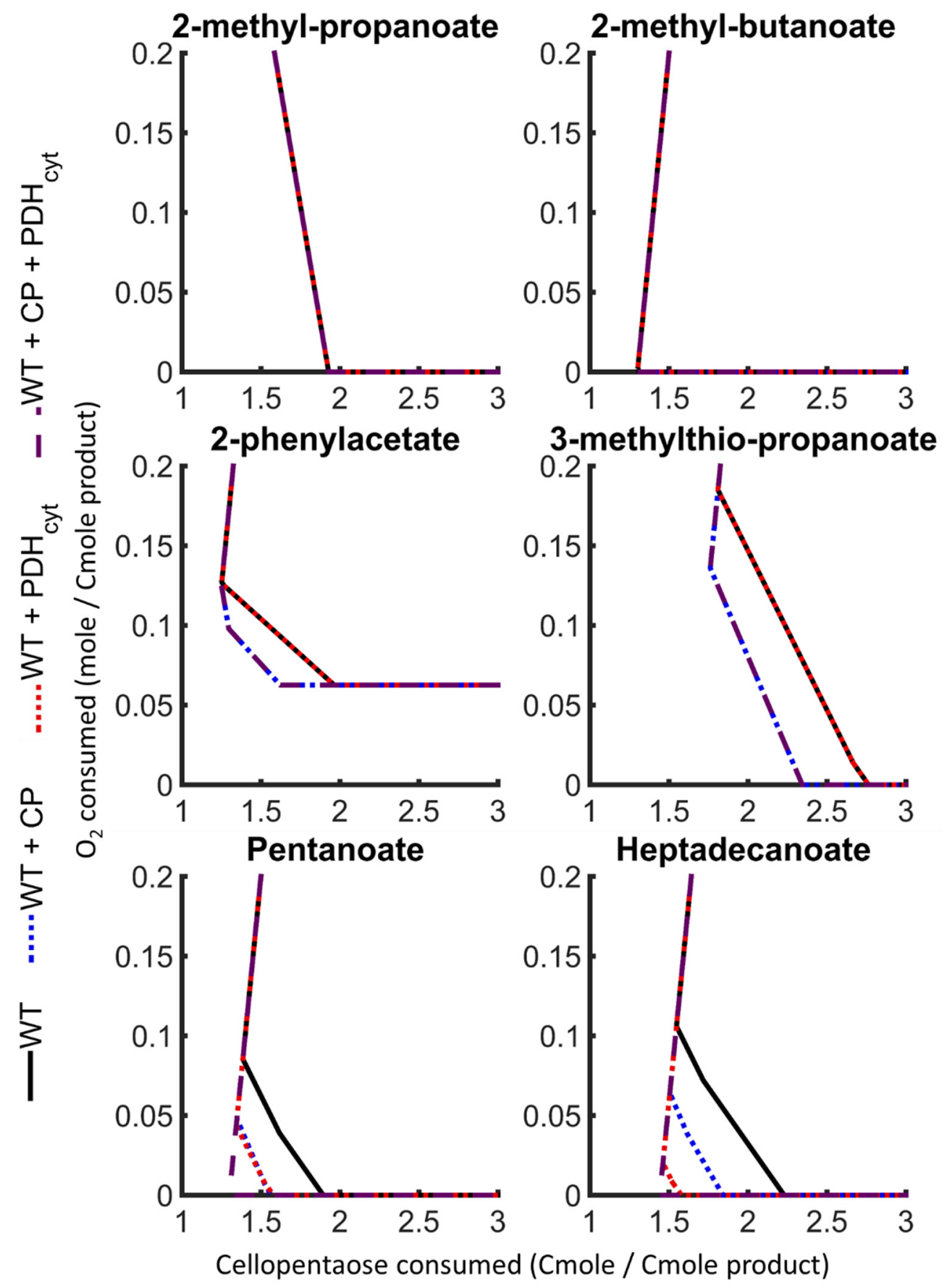

Figure 7. Theoretical effect of recombinant cellobiose phosphorylase $(\mathrm{CP})$ and cytosolic pyruvate dehydrogenase $\left(\mathrm{PDH}_{\text {cyt }}\right)$ activity on functionalized hydrocarbon production properties in $A$. sarcoides NRRL 50072 (black lines), with CP (blue dotted line), with $\mathrm{PDH}_{\text {cyt }}$ (red dotted line), and both (purple dashed line).

\section{Discussion}

Functionalized hydrocarbons (FHs) produced by the endophytic fungi $A$. sarcoides have theoretical yields governed by the cellular energy, carbon, and electrons they require for production. The presented analysis quantified the theoretical yields of $161 \mathrm{FHs}$ from cellulose-based carbon sources to understand their production for ecological or industrial purposes. The study identified the most efficient, theoretical production of the 161 different 
FHs based on criteria such as the conversion of a substrate, the number of Cmoles per product, and the presence of different secondary functional groups. The systems-based analysis identified FHs production properties that would not otherwise be obvious. Noteworthy FH production considerations in a eukaryotic host include: (1) Ehrlich pathway-derived FHs are generally more efficient at retaining substrate electrons and Cmoles than fatty acid-derived FHs. However, Ehrlich pathway derivatives were generally less reduced compounds. (2) Aromatic, Ehrlich pathway derived FHs required the least amount of carbon source under aerobic conditions of any FH considered in the study. (3) Contrary to the production of small primary fermentation products, including acetate, ethanol, and lactate, $\mathrm{O}_{2}$ limitation decreased the theoretical yield of FHs due to energy limitations. (4) FHs produced from isopentyl derivatives were less sensitive to $\mathrm{O}_{2}$ limitation than aromatic derivatives.

Fatty acid production can account for the most commonly reported FHs from $A$. sarcoides (Table 1). The presented stoichiometric model examined the effect of different precursors, including common central carbon metabolism (i.e., acetyl-CoA) and amino acid-derived intermediates (i.e., propanoyl-CoA). These two initiating molecules impacted the production of FHs through electron and energy balances. For example, the production of propanoyl-CoA required more electrons than acetyl-CoA, thereby serving as an effective electron sink, reducing the production of primary fermentation products like acetate and ultimately improving carbon retention during $\mathrm{FH}$ production.

Genetic modifications were examined in silico that impacted energy conservation and the transport and compartmentalization of acetyl-CoA. Modification of enzymatic activity (i.e., $\mathrm{CP}$ and $\mathrm{PDH}_{\text {cyt }}$ ) was predicted to reduce the carbon source requirements of FH production to the theoretical limits. The stoichiometric analysis predicted the addition of $\mathrm{CP}$ and $\mathrm{PDH}_{\text {cyt }}$ activities to $\mathrm{FH}$ producing cells could increase $\mathrm{FH}$ yields up to $38 \%$ on the carbon source (Table 2). CP activity reduced the activation cost of the carbon source, and $\mathrm{PDH}_{\text {cyt }}$ activity reduced the energetic requirement for cytosolic acetyl-CoA, increasing the yield of FHs. The combined impact of these modifications reduced the requirement for carbon sources to produce fatty acid derivatives to $1.5 \mathrm{Cmoles}$ carbon source per Cmole of $\mathrm{FH}$. These modifications and others, such as disrupting acetyl-CoA partitioning between the mitochondria and cytosol, have increased product yields and rates in other genetically modified organisms [22,46].

In vitro analysis of $A$. sarcoides NRRL 50072 cultures were performed under different $\mathrm{O}_{2}$ partial pressures to further interpret in silico results (Supplementary Materials). The in vitro data supported predictions that cellular energy yields and, therefore, biomass yields would decrease with decreasing $\mathrm{O}_{2}$ availability (Figures S1 and S2). As the $\mathrm{O}_{2}$ partial pressure decreased, secretion of the primary fermentation products, acetate and ethanol, increased to balance cellular reducing equivalents and cellular energy production. In vitro changes in $A$. sarcoides growth and excreted products suggest that the transition to fermentative metabolism occurred when the headspace was less than $\sim 10 \% \mathrm{O}_{2}$ (Figure S1). The experimental yield of FHs produced per $\mathrm{g}$ of carbon source consumed did not change substantially with decreasing $\mathrm{O}_{2}$ partial pressures, but the diversity of secreted FHs increased (Table S1). All in vivo FH yields were at least $10^{4}$-fold lower than theoretical limits, which indicated production was not for catabolic purposes but rather served other biological functions, like perhaps, communication molecules. Feedback mechanisms in metabolism and physiology, such $\mathrm{O}_{2}$ concentration and carbon source, may control the production of specific FHs in vivo [47,48]. Previous reports indicate that some plant tissues also have metabolic shifts at $\sim 10 \% \mathrm{O}_{2}$ partial pressure [49]. This may provide an in vivo feedback mechanism indicating an infected host, thereby triggering increased production of defensive compounds from its endophytic symbionts. For example, at $\sim 21 \% \mathrm{O}_{2}$ partial pressure, a common signaling molecule for hyphal growth, 1,3-octadiene, was observed. In contrast, more reduced biomass derivatives, such as Ehrlich pathway derivatives, were observed with decreased $\mathrm{O}_{2}$ partial pressure (Table S1), suggesting a communication role linked to intracellular redox state. 


\section{Conclusions}

Biological FH synthesis is metabolically costly, yet it is observed in many microorganisms spanning many ecosystems. The current study presents the most extensive stoichiometric analysis of FHs produced by either an endophytic or filamentous fungus. The results provide metabolic blueprints for producing 161 different FHs, quantifying the metabolic costs in terms of cellulose-based carbon source and $\mathrm{O}_{2}$ to produce each FH. The simulations also provide insights into natural conditions that would favor their production and provide targets for metabolic engineering of eukaryotic hosts to increase FH production efficiency.

Supplementary Materials: The following are available online at https://www.mdpi.com/article/ 10.3390/jof7060435/s1, Figure S1: Observed product yields from A. sarcoides NRRL 50072 as a function of the initial $\mathrm{O}_{2}$ concentration in the headspace, Table S1: Observed products of $A$. sarcoides NRRL 50072 cultivation under varying initial $\mathrm{O}_{2}$ partial pressure, Figure S2: Biomass production under varying initial $\mathrm{O}_{2}$ partial pressures, and associated files detailing figure construction and the metabolic model.

Author Contributions: Conceptualization, K.A.H., N.D.M., B.M.P., and R.P.C.; methodology, K.A.H., N.D.M., B.M.P., and R.P.C.; formal analysis, K.A.H., N.D.M., B.M.P., and R.P.C.; investigation, K.A.H. and N.D.M.; resources, B.M.P. and R.P.C.; data curation, K.A.H. and N.D.M.; writing-original draft preparation, K.A.H., N.D.M., B.M.P., and R.P.C.; writing-review and editing, K.A.H., N.D.M., B.M.P., and R.P.C.; visualization, K.A.H., N.D.M., B.M.P., and R.P.C.; supervision, B.M.P. and R.P.C.; project administration, B.M.P. and R.P.C.; funding acquisition, B.M.P. and R.P.C. All authors have read and agreed to the published version of the manuscript.

Funding: This research was funded by the National Science Foundation, Emerging Frontiers in Research and Innovation, grant number 093761.

Institutional Review Board Statement: Not applicable.

Data Availability Statement: All data are provided in the Supplementary Materials.

Acknowledgments: Our appreciation goes to Albert Parker for help with statistical analyses.

Conflicts of Interest: The authors declare no conflict of interest. The funders had no role in the design of the study; in the collection, analyses, or interpretation of data; in the writing of the manuscript, or in the decision to publish the results.

\section{References}

1. Strobel, G.A.; Knighton, B.; Kluck, K.; Ren, Y.; Livinghouse, T.; Griffin, M.A.; Spakowicz, D.J; Sears, J. The production of myco-diesel hydrocarbons and their derivatives by the endophytic fungus Gliocladium roseum (NRRL 50072). Microbiology 2008, 154, 3319-3328. [CrossRef] [PubMed]

2. Griffin, M.A.; Spakowicz, D.J.; Gianoulis, T.A.; Strobel, S.A. Volatile organic compound production by organisms in the genus Ascocoryne and a re-evaluation of myco-diesel production by NRRL 50072. Microbiology 2010, 156, 3814-3829. [CrossRef] [PubMed]

3. Gianoulis, T.A.; Griffin, M.A.; Spakowicz, D.J.; Dunican, B.F.; Alpha, C.J.; Sboner, A.; Michael Sismour, A.; Kodira, C.; Egholm, M.; Church, G.M.; et al. Genomic analysis of the hydrocarbon-producing, cellulolytic, endophytic fungus Ascocoryne sarcoides. PLoS Genet. 2012, 8, e1002558. [CrossRef]

4. Mallette, N.D.; Knighton, B.; Strobel, G.A.; Carlson, R.P.; Peyton, B.M. Resolution of volatile fuel compound profiles from Ascocoryne sarcoides: A comparison by proton transfer reaction-mass spectrometry and solid phase microextraction gas chromatographymass spectrometry. AMB Express 2012, 2, 23. [CrossRef] [PubMed]

5. Mallette, N.; Pankratz, E.M.; Parker, A.E.; Strobel, G.A.; Busse, S.C.; Carlson, R.P.; Peyton, B.M. Evaluation of cellulose as a substrate for hydrocarbon fuel production by Ascocoryne sarcoides (NRRL 50072). J. Sustain. Bioenergy Syst. 2014, 04, 33-49. [CrossRef]

6. Stinson, M.; Ezra, D.; Hess, W.M.; Sears, J.; Strobel, G. An endophytic Gliocladium sp. of Eucryphia cordifolia producing selective volatile antimicrobial compounds. Plant Sci. 2003, 165, 913-922. [CrossRef]

7. Hussa, E.a.; Goodrich-Blair, H. It takes a village: Ecological and fitness impacts of multipartite mutualism. Annu. Rev. Microbiol. 2013, 67, 161-178. [CrossRef] [PubMed]

8. Strobel, G.A.; Daisy, B.; Castillo, U.F.; Harper, J. Natural products from endophytic microorganisms. J. Nat. Prod. 2004, 67, 257-268. [CrossRef] 
9. Verma, V.C.; Kharwar, R.N.; Strobel, G.A. Chemical and functional diversity of natural products from plant associated endophytic fungi. Nat. Prod. Commun. 2009, 4, 1511-1532. [CrossRef]

10. Yan, Q.; Pfleger, B.F. Revisiting metabolic engineering strategies for microbial synthesis of oleochemicals. Metab. Eng. 2020, 58, 35-46. [CrossRef]

11. Zhou, Y.J.; Hu, Y.; Zhu, Z.; Siewers, V.; Nielsen, J. Engineering 1-Alkene Biosynthesis and Secretion by Dynamic Regulation in Yeast. ACS Synth. Biol. 2018, 7, 584-590. [CrossRef] [PubMed]

12. Yan, Q.; Simmons, T.R.; Cordell, W.T.; Hernández Lozada, N.J.; Breckner, C.J.; Chen, X.; Jindra, M.A.; Pfleger, B.F. Metabolic engineering of $\beta$-oxidation to leverage thioesterases for production of 2-heptanone, 2-nonanone and 2-undecanone. Metab. Eng. 2020, 61, 335-343. [CrossRef] [PubMed]

13. Flores, A.; Wang, X.; Nielsen, D.R. Recent trends in integrated bioprocesses: Aiding and expanding microbial biofuel/biochemical production. Curr. Opin. Biotechnol. 2019, 57, 82-87. [CrossRef]

14. Schulz, S.; Dickschat, J.S. Bacterial volatiles: The smell of small organisms. Nat. Prod. Rep. 2007, 24, 814-842. [CrossRef]

15. Hazelwood, L.A.; Daran, J.-M.; van Maris, A.J.A.; Pronk, J.T.; Dickinson, J.R. The Ehrlich pathway for fusel alcohol production: A century of research on Saccharomyces cerevisiae metabolism. Appl. Environ. Microbiol. 2008, 74, 2259-2266. [CrossRef]

16. Tejero Rioseras, A.; Garcia Gomez, D.; Ebert, B.E.; Blank, L.M.; Ibáñez, A.J.; Sinues, P.M.-L. Comprehensive Real-Time Analysis of the Yeast Volatilome. Sci. Rep. 2017, 7, 14236. [CrossRef]

17. Lee, J.-W.; Trinh, C.T. Towards renewable flavors, fragrances, and beyond. Curr. Opin. Biotechnol. 2020, 61, 168-180. [CrossRef]

18. Xu, P.; Qiao, K.; Ahn, W.S.; Stephanopoulos, G. Engineering Yarrowia lipolytica as a platform for synthesis of drop-in transportation fuels and oleochemicals. Proc. Natl. Acad. Sci. USA 2016, 113, 10848-10853. [CrossRef]

19. Tehlivets, O.; Scheuringer, K.; Kohlwein, S.D. Fatty acid synthesis and elongation in yeast. Biochim. Biophys. Acta 2007, 1771, 255-270. [CrossRef]

20. Carlson, R.; Fell, D.; Srienc, F. Metabolic pathway analysis of a recombinant yeast for rational strain development. Biotechnol. Bioeng. 2002, 79, 121-134. [CrossRef] [PubMed]

21. Beopoulos, A.; Cescut, J.; Haddouche, R.; Uribelarrea, J.-L.; Molina-Jouve, C.; Nicaud, J.-M. Yarrowia lipolytica as a model for bio-oil production. Prog. Lipid Res. 2009, 48, 375-387. [CrossRef] [PubMed]

22. Blazeck, J.; Hill, A.; Liu, L.; Knight, R.; Miller, J.; Pan, A.; Otoupal, P.; Alper, H.S. Harnessing Yarrowia lipolytica lipogenesis to create a platform for lipid and biofuel production. Nat. Commun. 2014, 5, 3131. [CrossRef]

23. Hynes, M.J.; Murray, S.L. ATP-citrate lyase is required for production of cytosolic acetyl coenzyme A and development in Aspergillus nidulans. Eukaryot. Cell 2010, 9, 1039-1048. [CrossRef] [PubMed]

24. Gu, C.; Kim, G.B.; Kim, W.J.; Kim, H.U.; Lee, S.Y. Current status and applications of genome-scale metabolic models. Genome Biol. 2019, 20, 121. [CrossRef] [PubMed]

25. Poolman, M.G.; Miguet, L.; Sweetlove, L.J.; Fell, D.A. A Genome-Scale Metabolic Model of Arabidopsis and Some of Its Properties. Plant Physiol. 2009, 151, 1570-1581. [CrossRef] [PubMed]

26. Cheung, C.Y.M.; Williams, T.C.R.; Poolman, M.G.; Fell, D.A.; Ratcliffe, R.G.; Sweetlove, L.J. A method for accounting for maintenance costs in flux balance analysis improves the prediction of plant cell metabolic phenotypes under stress conditions. Plant J. 2013, 75, 1050-1061. [CrossRef]

27. Moejes, F.W.; Matuszyńska, A.; Adhikari, K.; Bassi, R.; Cariti, F.; Cogne, G.; Dikaios, I.; Falciatore, A.; Finazzi, G.; Flori, S.; et al. A systems-wide understanding of photosynthetic acclimation in algae and higher plants. J. Exp. Bot. 2017, 68, 2667-2681. [CrossRef]

28. Klamt, S.; Stelling, J. Two approaches for metabolic pathway analysis? Trends Biotechnol. 2003, 21, 64-69. [CrossRef]

29. Orth, J.D.; Thiele, I.; Palsson, B.Ø. What is flux balance analysis? Nat. Biotechnol. 2010, 28, 245-248. [CrossRef] [PubMed]

30. Reed, J.L.; Palsson, B.O. Thirteen years of building constraint-based in silico models of Escherichia coli. J. Bacteriol. 2003, 185, 2692-2699. [CrossRef]

31. Trinh, C.T.; Wlaschin, A.; Srienc, F. Elementary mode analysis: A useful metabolic pathway analysis tool for characterizing cellular metabolism. Appl. Microbiol. Biotechnol. 2009, 81, 813-826. [CrossRef] [PubMed]

32. Varma, A.; Boesch, B.W.; Palsson, B.O. Stoichiometric interpretation of Escherichia coli glucose catabolism under various oxygenation rates. Appl. Environ. Microbiol. 1993, 59, 2465-2473. [CrossRef]

33. Llaneras, F.; Picó, J. Which metabolic pathways generate and characterize the flux space? A comparison among elementary modes, extreme pathways and minimal generators. J. Biomed. Biotechnol. 2010, 2010, 753904. [CrossRef] [PubMed]

34. Schilling, C.H.; Letscher, D.; Palsson, B.Ø. Theory for the systemic definition of metabolic pathways and their use in interpreting metabolic function from a pathway-oriented perspective. J. Theor. Biol. 2000, 203, 229-248. [CrossRef] [PubMed]

35. Lu, Y.; Ye, C.; Che, J.; Xu, X.; Shao, D.; Jiang, C.; Liu, Y.; Shi, J. Genomic sequencing, genome-scale metabolic network reconstruction, and in silico flux analysis of the grape endophytic fungus Alternaria sp. MG1. Microb. Cell Fact. 2019, 18. [CrossRef]

36. Emanuelsson, O.; Nielsen, H.; Brunak, S.; von Heijne, G. Predicting subcellular localization of proteins based on their N-terminal amino acid sequence. J. Mol. Biol. 2000, 300, 1005-1016. [CrossRef]

37. Grube, M.; Zagreba, E.; Gromozova, E.; Fomina, M. Comparative investigation of the macromolecular composition of mycelia forms Thielavia terrestris by infrared spectroscopy. Vib. Spectrosc. 1999, 19, 301-306. [CrossRef]

38. Klamt, S.; Saez-Rodriguez, J.; Gilles, E.D. Structural and functional analysis of cellular networks with CellNetAnalyzer. BMC Syst. Biol. 2007, 129, 329-351. [CrossRef] 
39. Klamt, S.; von Kamp, A. An application programming interface for CellNetAnalyzer. Biosystems 2011, 105, 162-168. [CrossRef] [PubMed]

40. Jungreuthmayer, C.; Ruckerbauer, D.E.; Zanghellini, J. regEfmtool: Speeding up elementary flux mode calculation using transcriptional regulatory rules in the form of three-state logic. Biosystems 2013, 113, 37-39. [CrossRef]

41. Schellenberger, J.; Que, R.; Fleming, R.M.T.; Thiele, I.; Orth, J.D.; Feist, A.M.; Zielinski, D.C.; Bordbar, A.; Lewis, N.E.; Rahmanian, S.; et al. Quantitative prediction of cellular metabolism with constraint-based models: The COBRA Toolbox v2.0. Nat. Protoc. 2011, 6, 1290-1307. [CrossRef]

42. Minty, J.J.; Singer, M.E.; Scholz, S.a.; Bae, C.-H.H.; Ahn, J.-H.H.; Foster, C.E.; Liao, J.C.; Lin, X.N. Design and characterization of synthetic fungal-bacterial consortia for direct production of isobutanol from cellulosic biomass. Proc. Natl. Acad. Sci. USA 2013, 110, 14592-14597. [CrossRef] [PubMed]

43. Schirmer, A.; Rude, M.A.; Li, X.; Popova, E.; del Cardayre, S.B. Microbial biosynthesis of alkanes. Science 2010, 329, 559-562. [CrossRef]

44. Ladygina, N.; Dedyukhina, E.G.; Vainshtein, M.B. A review on microbial synthesis of hydrocarbons. Process Biochem. 2006, 41, 1001-1014. [CrossRef]

45. Carlson, R.; Srienc, F. Fundamental Escherichia coli biochemical pathways for biomass and energy production: Creation of overall flux states. Biotechnol. Bioeng. 2004, 86, 149-162. [CrossRef] [PubMed]

46. Runguphan, W.; Keasling, J.D. Metabolic engineering of Saccharomyces cerevisiae for production of fatty acid-derived biofuels and chemicals. Metab. Eng. 2014, 21, 103-113. [CrossRef]

47. Schoen, H.R.; Hunt, K.A.; Strobel, G.A.; Peyton, B.M.; Carlson, R.P. Carbon chain length of biofuel- and flavor-relevant volatile organic compounds produced by lignocellulolytic fungal endophytes changes with culture temperature. Mycoscience 2017, 58 . [CrossRef]

48. Schoen, H.R.; Knighton, W.B.; Peyton, B.M. Endophytic fungal production rates of volatile organic compounds are highest under microaerophilic conditions. Microbiology 2017, 163, 1767-1777. [CrossRef]

49. Gupta, K.J.; Zabalza, A.; van Dongen, J.T. Regulation of respiration when the oxygen availability changes. Physiol. Plant. 2009, 137, 383-391. [CrossRef] 